\title{
Preliminary comparison between real-time in-vivo spectral and transverse oscillation velocity estimates
}

Pedersen, Mads Møller; Pihl, Michael Johannes; Haugaard, Per; Hansen, Jens Munk; Hansen, Kristoffer Lindskov; Nielsen, Michael Bachmann; Jensen, Jørgen Arendt

\section{Published in:}

Proceedings of SPIE

Link to article, DOI:

10.1117/12.877221

Publication date:

2011

Link back to DTU Orbit

Citation (APA):

Pedersen, M. M., Pihl, M. J., Haugaard, P., Hansen, J. M., Hansen, K. L., Nielsen, M. B., \& Jensen, J. A. (2011). Preliminary comparison between real-time in-vivo spectral and transverse oscillation velocity estimates. In $\mathrm{J}$. D'hooge, \& M. M. Doyley (Eds.), Proceedings of SPIE (Vol. 7968). SPIE - International Society for Optical Engineering. https://doi.org/10.1117/12.877221

\section{General rights}

Copyright and moral rights for the publications made accessible in the public portal are retained by the authors and/or other copyright owners and it is a condition of accessing publications that users recognise and abide by the legal requirements associated with these rights.

- Users may download and print one copy of any publication from the public portal for the purpose of private study or research.

- You may not further distribute the material or use it for any profit-making activity or commercial gain

- You may freely distribute the URL identifying the publication in the public portal 


\title{
Preliminary comparison between real-time in vivo Spectral and Transverse Oscillation velocity estimates
}

\author{
Mads Møller Pedersen ${ }^{1}$, Michael Johannes Pihl ${ }^{2}$, Per Haugaard ${ }^{3}$, Jens Munk Hansen², \\ Kristoffer Lindskov Hansen ${ }^{1}$, Michael Bachmann Nielsen ${ }^{1}$, Jørgen Arendt Jensen² \\ ${ }^{1}$ Dept. of Radiology, Rigshospitalet, 2100 Copenhagen, Denmark \\ ${ }^{2}$ Center for Fast Ultrasound Imaging, Dept. of Elec. Eng., Technical University of Denmark, \\ 2800 Lyngby, Denmark \\ ${ }^{3}$ B-K Medical A/S, Mileparken 34, 2730 Herlev, Denmark
}

\begin{abstract}
1. ABSTRACT
Spectral velocity estimation is considered the gold standard in medical ultrasound. Peak systole (PS), end diastole (ED), and resistive index (RI) are used clinically. Angle correction is performed using a flow angle set manually. With Transverse Oscillation (TO) velocity estimates the flow angle, peak systole $\left(\mathrm{PS}_{T O}\right)$, end diastole $\left(\mathrm{ED}_{T O}\right)$, and resistive index $\left(\mathrm{RI}_{T O}\right)$ are estimated. This study investigates if these clinical parameters are estimated equally good using spectral and TO data. The right common carotid arteries of three healthy volunteers were scanned longitudinally. Average TO flow angles and std were calculated $\{52 \pm 18 ; 55 \pm 23$; $60 \pm 16\}^{\circ}$. Spectral angles $\{52 ; 56 ; 52\}^{\circ}$ were obtained from the B-mode images. Obtained values are: $\mathrm{PS}_{T O}$ $\{76 \pm 15 ; 89 \pm 28 ; 77 \pm 7\} \mathrm{cm} / \mathrm{s}$, spectral PS $\{77 ; 110 ; 76\} \mathrm{cm} / \mathrm{s}, \operatorname{ED}_{T O}\{10 \pm 3 ; 14 \pm 8 ; 15 \pm 3\} \mathrm{cm} / \mathrm{s}$ spectral ED $\{18 ; 13 ; 20\} \mathrm{cm} / \mathrm{s}, \mathrm{RI}_{T O}\{0.87 \pm 0.05 ; 0.79 \pm 0.21 ; 0.79 \pm 0.06\}$, and spectral RI $\{0.77 ; 0.88$ $; 0.73\}$. Vector angles are within \pm two std of the spectral angle. TO velocity estimates are within \pm three std of the spectral estimates. $\mathrm{RI}_{T O}$ are within \pm two std of the spectral estimates. Preliminary data indicates that the TO and spectral velocity estimates are equally good. With TO there is no manual angle setting and no flow angle limitation. TO velocity estimation can also automatically handle situations where the angle varies over the cardiac cycle. More detailed temporal and spatial vector estimates with diagnostic potential are available with the TO velocity estimation.
\end{abstract}

Keywords: Ultrasound imaging, transverse oscillation, vector flow, blood velocity, spectral estimation, in vivo

\section{INTRODUCTION}

\subsection{Spectral velocity estimation}

Clinical evaluations of arterial blood velocities are widely used. Today the only clinically available method is spectral velocity estimation. The peak systolic and the end diastolic velocities are estimated and can be used to calculate the resistive index. A spectrogram shows the temporal distribution of the blood velocities. The resistive index of a feeding artery to an organ is used to evaluate the inflow resistance. ${ }^{1}$ Measurements on the renal artery is used to diagnose the presence of renal artery stenosis. ${ }^{2}$ Both peak velocity and RI is increased and the spectrogram waveform is changed. ${ }^{2,3}$ Identical changes in the pulmonary arteries are used to identify pulmonary hypertension. ${ }^{4}$ Velocity changes of the portal vein are also used to identify restrictive liver conditions such as cirrhosis and autoimmune hepatitis. ${ }^{5,6}$ In cardiology the velocity estimates are used to estimate pressure gradients of the aortic valve. ${ }^{7,8}$ The spectrogram waveform and the resistive index of the umbilical artery is used to evaluate the presence of increased resistance. Velocity estimation of the fetal aorta is also evaluated to diagnose congenital conditions. ${ }^{9}$

Further author information: (Send correspondence to Mads Møller Pedersen) Mads Møller Pedersen: E-mail: phd@medit.dk, Phone: +45 35453545

Michael Johannes Pihl: E-mail: mjp@elektro.dtu.dk, Jens Munks Hansen: E-mail: jmh@elektro.dtu.dk, Per Haugaard: E-mail: peh@bkmed.dk, Michael Bachmann Nielsen: E-mail: mbn@dadl.dk, Jørgen Arendt Jensen: E-mail: jaj@elektro.dtu.dk 


\section{Limitations}

Spectral estimation obtains velocities only in the axial direction. ${ }^{10,11}$ However, the angle of the flow is rarely aligned along the ultrasound beam. The axial velocities, $v_{z}$, can be angle corrected by using the flow angle, $\theta$, in the cosine relation $(1) \cdot{ }^{10,11}$

$$
v=\frac{v_{z}}{\cos \theta}
$$

With spectral estimation the flow angle is set manually by the sonographer from the B-mode image (see Fig. 1). The flow angle must be below $60^{\circ}$ for the corrected velocity estimate to be accurate assuming the angle is fixed over the cardiac cycle. The range gate position and width is set manually by the sonographer using the B-mode image. The width of the range gate affects the velocity estimation. The sonographer can select the entire vessel diameter or only a small area of the vessel lumen.

Real-time calculations of the peak systole, end diastole, and the resistive index are performed. The PS and ED values are calculated from angle corrected axial velocities. Only one angle is used and only one set of estimates are achieved with spectral estimation. During the data acquisition the tissue moves due to pulse and respiratory movements. No motion compensation is available for the range gate to follow the moving vessel. Therefore, the acquired data are easily distorted by vessel movement.

\subsection{Transverse Oscillation}

There are two main advantages of TO: No angle correction and no angle limitation. Both axial and transverse velocities are obtained. ${ }^{12}$ Angle correction is not needed as the blood flow velocity is calculated from the axial and transverse components. As the transverse component is available flow perpendicular to the transducer surface is also obtained. Therefore, TO can measure blood velocities in all directions. ${ }^{13,14}$

\section{Limitations}

At present the clinical TO implementation only works with one transducer type. As it is linear the penetration depth is limited to $4-5 \mathrm{~cm}$. Therefore, the clinical use is still limited as the renal artery, portal vein, heart and fetus scanning requires a convex transducer with a penetration depth of 10-14 $\mathrm{cm}$. The common carotid artery was selected as it is superficial and easy to locate with the linear transducer.

\subsection{Purpose}

The purpose of this preliminary in vivo study is to compare blood flow angle and velocity estimates using spectral estimation and TO. Velocity estimation of the common carotid artery is widely used and easily obtained. Therefore this measurement was selected to compare the conventional spectral velocity estimation, and the TO technique. The blood flow angle, peak systole velocities, end diastole velocities, and resistive indexes are compared. With simultaneous data acquisition, the velocity estimates of the two methods are comparable as they do not differ in time. This study presents the first real-time, in vivo data using a commercial implementation of TO without any use of contrast enhancing agents. In this paper the techniques are introduced and the study setup, data calculation, and results are presented followed by a discussion and a conclusion. 


\section{MATERIALS AND METHODS}

\subsection{Data acquisition}

Three healthy volunteers of ages 34, 35, and 39 years participated in this study. A commercial ultrasound scanner (ProFocus 2202, B-K Medical) and a 7.6 MHz linear transducer was used (Type: 8670, B-K Medical, Denmark). The right common carotid artery of each volunteer was scanned longitudinally. A scan mode allowing simultaneous data acquisition for spectral velocity estimation and TO was used. The scan setting was optimized for spectral velocity estimation. In the longitudinal plane the center of the artery was located. The angle between the ultrasound beam and the blood flow direction (the blood flow angle) was kept below $60^{\circ}$. This was achieved by tilting the transducer. The selected scan mode did not allow tilting of the beam. The range gate was set to cover the entire vessel lumen (Fig. 1). A maximum scan depth of $2.8 \mathrm{~cm}$ was selected. The maximum frame rate allowed by the frame buffer in the scanner was 14.8 frames per second. Thus, an avi video sequence of 166 frames covering 11.2 seconds were obtained for each volunteer.

\section{Transverse Oscillation settings}

A maximum of 16 shots per estimate, a medium 2D filter, a TO gain of 50\%, and a center frequency of $5 \mathrm{MHz}$ was used. No persistence was used. For each volunteer, the pulse repetition frequency was adjusted to avoid aliasing. The wall filter was adjusted to avoid artifacts from movements of the surrounding tissue. Identical PRF and wall filter values were set for spectral and TO data (Table 5). The color box for TO data acquisition was set to cover the range gate area.

\section{Spectral velocity settings}

The PRF and the wall filter were set identical to the TO. A gain of $50 \%$ was set. No smoothing filter was used. Angle correction was set visually by the sonographer. Real-time calculation of the PS, ED, and RI was selected.

\subsection{Data calculation}

\section{Transverse Oscillation data}

TO data was encoded as colored pixels. Using a Matlab script and a circular color map (Fig. 2) the color data was decoded into vector data. Thus, each pixel in the range gate area was represented by a vector with an axial and a transverse component. The resulting velocity, $v_{T O}$ is calculated using the axial, $v_{z}$, and transverse, $v_{x}$, vector components by

$$
v_{T O}=\sqrt{v_{x}^{2}+v_{z}^{2}}
$$

The flow angle, $\theta_{T O}$, is calculated using the velocity and the transverse component the flow angle as

$$
\theta_{T O}=\cos ^{-1} \frac{v_{z}}{v_{T O}} .
$$

The pixel with the maximum velocity for each frame was selected for further analysis (Fig. 3). The average blood flow angle was calculated as the average flow angle in the range gate area throughout each sequence. The average peak value was calculated as the TO Peak Systole value for each volunteer. The lowest value before the peak was used to calculate the average TO End Diastole value. TO Resistive Index was calculated by

$$
R I_{T O}=\frac{P S_{T O}-E D_{T O}}{P S_{T O}} .
$$

\section{Spectral velocity data}

The maximum spectral velocity estimates were used for continuous calculation of the PS, ED, and RI. Calculations from the last frame of each sequence were obtained. These estimates represent the average values for the past eight seconds of spectral data (Fig. 1). 


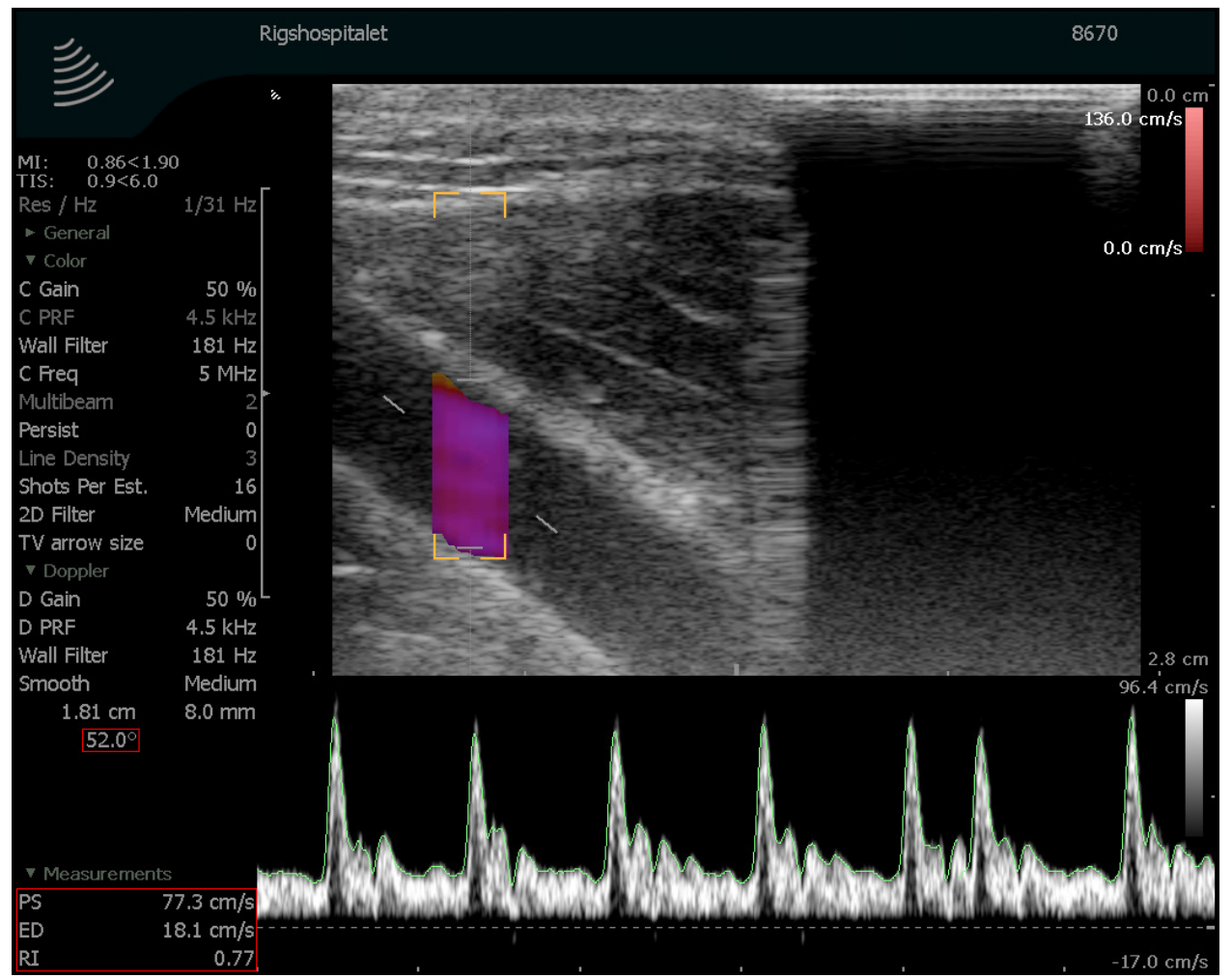

Figure 1: Simultaneous data acquisition using spectral estimation and Transverse Oscillation. The manually set flow angle and the spectral estimation results are marked in red at the lower left of the image. 

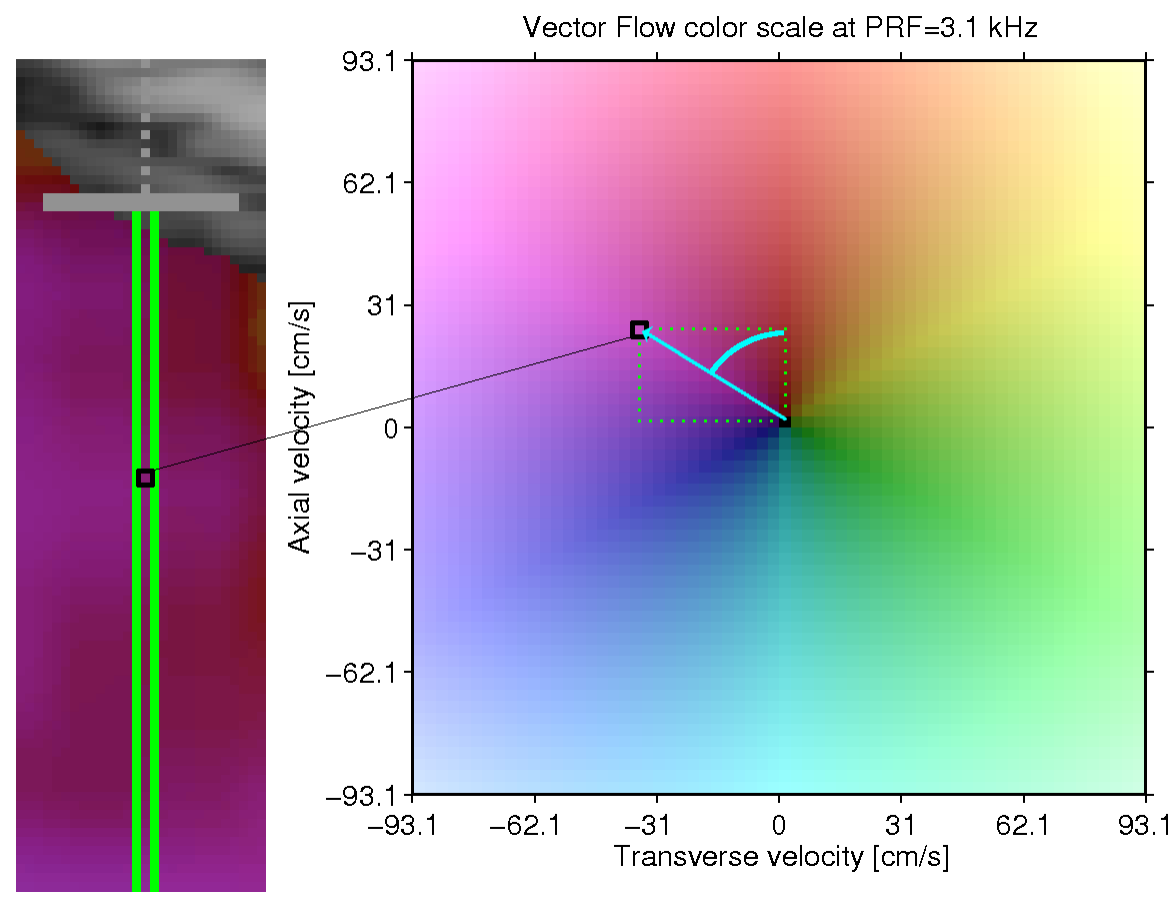

Figure 2: The range gate covering the whole vessel diameter. The pixels of the central line is used for spectral and TO estimates. The color values of each pixel contains the encoded vector information used for the TO method.

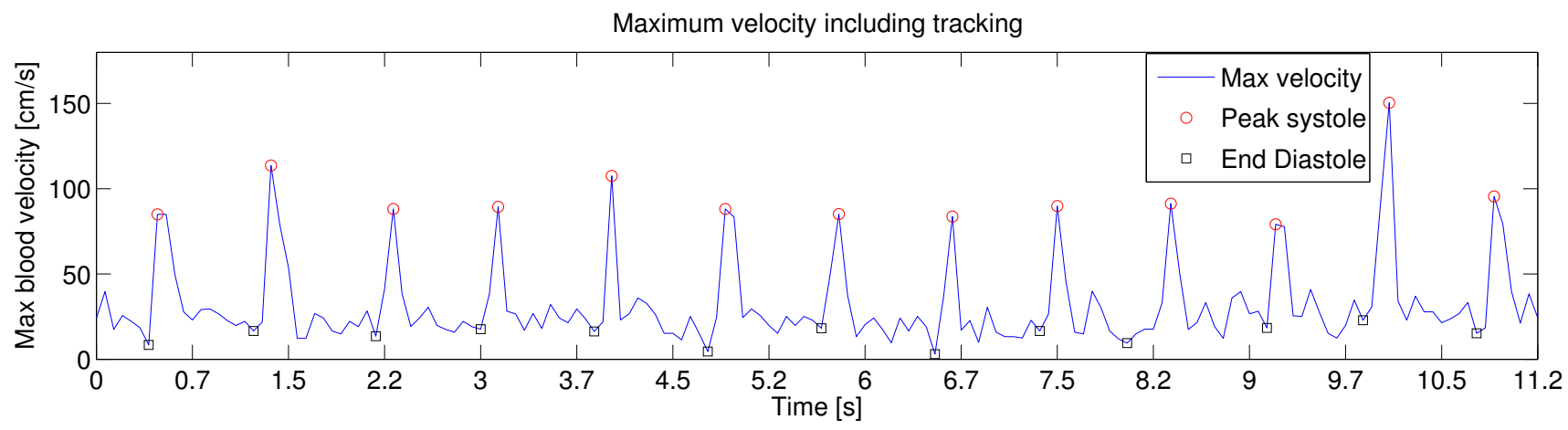

Figure 3: TO data. The maximum velocity estimates for each frame peak at the peak systole for the spectral estimate (red circles). 


\section{RESULTS}

Spectral flow angle and velocity estimation of PS, ED, and RI were obtained from the last frame in the avi sequences for each volunteer. Transverse Oscillation color data were converted into vector data and the average flow angle, the $\mathrm{PS}_{T O}, \mathrm{ED}_{T O}$, and $\mathrm{RI}_{T O}$ were calculated including the standard deviations. All results are shown in the tables below (Tables 1, 2, 3, 4). The PRF and Wall filter settings for each volunteer are listed in table 5 .

\begin{tabular}{|c|c|c|}
\hline & \multicolumn{2}{|c|}{ Blood flow angle $\left[^{\circ}\right]$} \\
\hline Data set & $\begin{array}{c}\text { Transverse } \\
\text { Oscillation }\end{array}$ & $\begin{array}{c}\text { Spectral } \\
\text { estimation }\end{array}$ \\
\hline 1 & $58 \pm 18$ & 52 \\
\hline 2 & $55 \pm 23$ & 56 \\
\hline 3 & $60 \pm 16$ & 52 \\
\hline
\end{tabular}

Table 1: Blood flow angles for TO and spectral estimation

\begin{tabular}{|c|c|c|}
\hline & \multicolumn{2}{|c|}{ Peak systole velocity $[\mathrm{cm} / \mathrm{s}]$} \\
\hline Data set & $\begin{array}{c}\text { Transverse } \\
\text { Oscillation }\end{array}$ & $\begin{array}{c}\text { Spectral } \\
\text { estimation }\end{array}$ \\
\hline 1 & $76 \pm 15$ & 77 \\
\hline 2 & $89 \pm 28$ & 110 \\
\hline 3 & $77 \pm 7$ & 77 \\
\hline
\end{tabular}

Table 2: Peak systole velocity for TO and spectral estimation

\begin{tabular}{|c|c|c|}
\hline & \multicolumn{2}{|c|}{ End diastole velocity $[\mathrm{cm} / \mathrm{s}]$} \\
\hline Data set & $\begin{array}{c}\text { Transverse } \\
\text { Oscillation }\end{array}$ & $\begin{array}{c}\text { Spectral } \\
\text { estimation }\end{array}$ \\
\hline 1 & $10 \pm 3$ & 18 \\
\hline 2 & $14 \pm 8$ & 13 \\
\hline 3 & $15 \pm 3$ & 21 \\
\hline
\end{tabular}

Table 3: End diastole for TO and spectral estimation

\begin{tabular}{|c|c|c|}
\hline & \multicolumn{2}{|c|}{ Resistive Index } \\
\hline Data set & $\begin{array}{c}\text { Transverse } \\
\text { Oscillation }\end{array}$ & $\begin{array}{c}\text { Spectral } \\
\text { estimation }\end{array}$ \\
\hline 1 & $0.87 \pm 0.05$ & 0.77 \\
\hline 2 & $0.79 \pm 0.21$ & 0.88 \\
\hline 3 & $0.79 \pm 0.06$ & 0.73 \\
\hline
\end{tabular}

Table 4: Resistive Index for TO and spectral estimation

\begin{tabular}{|c|c|c|}
\hline Volunteer & PRF $[\mathrm{kHz}]$ & Wall filter $[\mathrm{Hz}]$ \\
\hline 1 & 4.5 & 181 \\
\hline 2 & 7.1 & 143 \\
\hline 3 & 4.5 & 181 \\
\hline
\end{tabular}

Table 5: PRF and Wall filter settings used for TO and spectral estimation 


\section{DISCUSSION}

This study was optimized to favour the conventional method: spectral estimation. The scanner software made it possible to obtain simultaneous data with the two methods. However, one feature, tilting of the ultrasound beam, was not available. This feature is used in the clinical setting to keep the flow angle below $60^{\circ}$ without tilting the transducer on the skin. For this study the transducer was tilted to obtain a flow angle below $60^{\circ}$. This resulted in lack of data on the right side of the image (Fig. 1). The imaging of the common carotid artery was not affected.

The flow angle set by the sonographer lies within \pm one standard deviation of the average TO flow angle. No weighting of the flow angles according to velocity or position in the range gate area was performed. The use of a single flow angle with spectral velocity estimation is controversial. The PS lies within \pm one standard deviation of the $\mathrm{PS}_{T O}$ and the ED lies within \pm three standard deviations of the $\mathrm{ED}_{T O}$. The RI is within \pm two standard deviations of the $\mathrm{RI}_{T O}$. This indicates that this alternative blood velocity estimation method may be equally good at estimating PS, ED, and RI. A crucial advantage is that TO does not use angle correction. The subjective setting by the sonographer is, thus, removed from the estimation. With conventional spectral velocity estimation the analysis is performed in one line only. Clinical evaluation of the blood velocity before, over, and after a plaque formation is not possible. Such multi line analysis is not available in commercial scanners today. With TO a whole block of vector data is available. It only depends on the area covered by the color box (Fig. 1). Thus, estimation of multiple lines can be analysed with post processing the vector information. TO also works for angles close to $90^{\circ}$ contrary to traditional systems, where the angle has to be below $90^{\circ}$.

The range gate width is set by the sonographer. It is set to cover the area of the vessel lumen of interest. Simultaneous analysis of parts of the range gate, sub gates, is not possible with spectral estimation. The live calculation of PS, ED, and RI is performed on data from the entire range gate area and no sub gating is available commercially. Clinical investigation using the spatial and temporal flow angle information may reveal new information on blood flow near the vessel wall and in the center of the vessel.

The range gate does not change position if the tissue moves. Motion tracking of the vessel and adjustment of the range gate is not possible with conventional spectral estimation. Removing the artifacts due to respiratory movements and pulsation would be appreciated. No motion tracking is available with spectral estimation. With TO the data for such motion tracking is available. It should be investigated further with the present study setup.

The live spectral velocity estimations provide updated PS, ED, and RI values. With TO data a temporal post processing analysis was performed. Combined with the spatial information a sub gate at the vessel wall can be compared to a sub gate in the center of the vessel. Such dynamic comparison may provide further detailed information on the wall shear stress.

Aliasing is easily detected by the waveform of the spectrogram. As the TO technique may alias at PRF values acceptable for spectral estimation the identification of TO aliasing is important. The present scanner software shows TO aliasing by color code only. Thus, the sonographer has to watch for certain color changes to identify aliasing.

The frame rate of the avi sequences was limited to $14.8 \mathrm{~Hz}$, with a maximum length of $11.2 \mathrm{~s}$ due to limitations in the scanner frame grabber. As the peak velocities are present for a very short time it is possible that the estimated TO peak systole values are not representing the true peak values. It may have appeared in between two frames. Thus, the $\mathrm{PS}_{T O}$ may be lower than the true peak systolic velocity. However, the $\mathrm{PS}_{T O}$ is not lower than the spectral PS estimations.

Increasing the frame rate would require changes in the scanner software and hardware. Increasing the temporal resolution will increase the precision of the TO estimates. Increased recording time (sequence length) of 2-3 minutes instead of $11.2 \mathrm{~s}$ would make it possible to analyse phenomenas such as dose-response of drugs affecting the blood pressure and blood velocity. 


\section{CONCLUSION}

Simultaneous data acquisition using two methods, spectral velocity estimation and Transverse Oscillation, was performed. From the comparison of the peak systole, end diastole, and resistive index estimates the TO estimation is equally good. TO does not rely on a flow angle determined subjectively by a sonographer. Furthermore, detailed temporal and spatial angle and velocity information is available with TO. It makes several new applications available. In conclusion Transverse Oscillation is indicated to be equally good compared to spectral velocity estimation for evaluating the common carotid artery. More data is needed for statistical evaluation of this indication.

\section{REFERENCES}

[1] Tublin, M. E., Bude, R. O., and Platt, J. F., "Review. the resistive index in renal Doppler sonography: where do we stand?," AJR Am J Roentgenol 180, 885-92 (Apr. 2003).

[2] Radermacher, J., Chavan, A., Bleck, J., Vitzthum, A., Stoess, B., Gebel, M. J., Galanski, M., Koch, K. M., and Haller, H., "Use of Doppler ultrasonography to predict the outcome of therapy for renal-artery stenosis.," N Engl J Med 344, 410-7 (Feb. 2001).

[3] Krumme, B., "Renal Doppler sonography-update in clinical nephrology.," Nephron Clin Pract 103(2), c24-8 (2006).

[4] Lanzarini, L., Fontana, A., Campana, C., and Klersy, C., "Two simple echo-Doppler measurements can accurately identify pulmonary hypertension in the large majority of patients with chronic heart failure.," J Heart Lung Transplant 24, 745-54 (June 2005).

[5] Görg, C., Riera-Knorrenschild, J., and Dietrich, J., "Pictorial review: Colour Doppler ultrasound flow patterns in the portal venous system.," Br J Radiol 75, 919-29 (Nov. 2002).

[6] Westra, S. J., Zaninovic, A. C., Vargas, J., Hall, T. R., Boechat, M. I., and Busuttil, R. W., "The value of portal vein pulsatility on duplex sonograms as a sign of portal hypertension in children with liver disease.," AJR Am J Roentgenol 165, 167-72 (July 1995).

[7] O'Boyle, M. K., Vibhakar, N. I., Chung, J., Keen, W. D., and Gosink, B. B., "Duplex sonography of the carotid arteries in patients with isolated aortic stenosis: imaging findings and relation to severity of stenosis.," AJR Am J Roentgenol 166, 197-202 (Jan. 1996).

[8] Jassal, D. S., Tam, J. W., Dumesnil, J. G., Giannoccaro, P. J., Jue, J., Pandey, A. S., Joyner, C. D., Teo, K. K., and Chan, K. L., "Clinical usefulness of tissue Doppler imaging in patients with mild to moderate aortic stenosis: a substudy of the aortic stenosis progression observation measuring effects of rosuvastatin study.," J Am Soc Echocardiogr 21, 1023-7 (Sept. 2008).

[9] Wood, M. M., Romine, L. E., Lee, Y. K., Richman, K. M., O’Boyle, M. K., Paz, D. A., Chu, P. K., and Pretorius, D. H., "Spectral Doppler signature waveforms in ultrasonography: a review of normal and abnormal waveforms.," Ultrasound Q 26, 83-99 (June 2010).

[10] Evans, D., [Doppler ultrasound: Physics, instrumentation, and clinical applications], John Wiley \& Sons (1989).

[11] Jensen, J., [Estimation of blood velocities using ultrasound: a signal processing approach], Cambridge Univ Pr (1996).

[12] Jensen, J. A. and Munk, P., "A new method for estimation of velocity vectors.," IEEE Trans Ultrason Ferroelectr Freq Control 45(3), 837-51 (1998).

[13] Hansen, K. L., Udesen, J., Oddershede, N., Henze, L., Thomsen, C., Jensen, J. A., and Nielsen, M. B., "In vivo comparison of three ultrasound vector velocity techniques to MR phase contrast angiography.," Ultrasonics 49, 659-67 (Dec. 2009).

[14] Udesen, J., Nielsen, M. B., Nielsen, K. R., and Jensen, J. A., "Examples of in vivo blood vector velocity estimation.," Ultrasound Med Biol 33, 541-8 (Apr. 2007). 